\title{
Effects of transmission-blocking vaccines simultaneously targeting pre- and post- fertilization antigens in the rodent malaria parasite Plasmodium yoelii
}

\author{
Li Zheng ${ }^{1}$, Wei Pang ${ }^{1}$, Zanmei Qi ${ }^{1}$, Enjie Luo ${ }^{2}$, Liwang Cui ${ }^{3}$ and Yaming Cao ${ }^{1 *}$
}

\begin{abstract}
Background: Transmission-blocking vaccine (TBV) is a promising strategy for interrupting the malaria transmission cycle. Current TBV candidates include both pre- and post-fertilization antigens expressed during sexual development of the malaria parasites.

Methods: We tested whether a TBV design combining two sexual-stage antigens has better transmission-blocking activity. Using the rodent malaria model Plasmodium yoelii, we pursued a DNA vaccination strategy with genes encoding the gametocyte antigen Pys48/45 and the major ookinete surface protein Pys 25.

Results: Immunization of mice with DNA constructs expression either Pys48/45 or Pys 25 elicited strong antibody responses, which specifically recognized a $\sim 45$ and $\sim 25 \mathrm{kDa}$ protein from gametocyte and ookinete lysates, respectively. Immune sera from mice immunized with DNA constructs expressing Pys48/45 and Pys 25 individually and in combination displayed evident transmission-blocking activity in in vitro ookinete culture and direct mosquito feeding experiments. With both assays, the Pys 25 sera had higher transmission-blocking activity than the Pys48/45 sera. Intriguingly, compared with the immunization with the individual DNA vaccines, immunization with both DNA constructs produced lower antibody responses against individual antigens. The resultant immune sera from the composite vaccination had significantly lower transmission-blocking activity than those from Pys 25 DNA immunization group, albeit the activity was substantially higher than that from the Pys48 DNA vaccination group.

Conclusions: This result suggested that vaccination with the two DNA constructs did not achieve a synergistic effect, but rather caused interference in inducing antigen-specific antibody responses. This result has important implications for future design of composite vaccines targeting different sexual antigens.
\end{abstract}

Keywords: Malaria, Plasmodium yoelii, Transmission-blocking vaccine, DNA vaccine, Pys25, Pys48/45

\section{Background}

Malaria is a devastating disease caused by malaria parasites in the genus Plasmodium. In nature, human malaria transmission requires Anopheles mosquitoes as obligate vectors. According to a recent WHO report, in 2015 it was estimated that there were 214 million cases of malaria globally (range: 149-303 million), leading to

\footnotetext{
* Correspondence: ymcao@mail.cmu.edu.cn

${ }^{1}$ Department of Immunology, College of Basic Medical Sciences, China Medical University, No.77 Puhe Road, Shenyang North New Area, Shenyang, Liaoning Province, People's Republic of China

Full list of author information is available at the end of the article
}

438,000 deaths (range: 236,000-635,000) [1]. Current tools for combating malaria include vector control with insecticides and artemisinin-based combination therapies $[2,3]$. The emergence and spread of drug-resistant parasites over the last four decades, especially with the recent detection of resistance against the front-line treatment artemisinins, highlight the necessity for new control strategies. In this regard, the development of a safe and effective antimalarial vaccine is expected to play an important role in integrated malaria control $[4,5]$.

Vaccine development efforts have focused on candidate antigens present in the pre-erythrocytic, erythrocytic and 
sexual stages of the parasites. Sexual stages of the malaria parasites are critical for transmission from humans to mosquitoes. During sexual development, male and female gametocytes in the peripheral blood rapidly differentiate into gametes upon uptake by an Anopheles vector. Following fertilization of the male and female gametes, zygotes are formed and develop into motile ookinetes. Ookinetes traverse the peritrophic matrix and midgut epithelium, lodge under the basal lamina of the midgut, and develop into oocysts [6]. A transmission-blocking vaccine (TBV) specifically targets the sexual development of the parasite in the mosquito vector and elicits immunity that effectively blocks transmission of the parasite from humans to mosquitoes [7].

To date, a number of TBV candidates have been investigated and only a handful of antigens show clear evidence of transmission-blocking (TB) activity, including P230, P48/45 and P25 and P28. Pfs48/45 is a prefertilization antigen and plays an essential role in parasite fertilization. Targeted disruption of the gene affects the male gamete's capacity to bind to female gametes [8], and antibodies targeting conformational epitopes of Pfs48/45 prevent fertilization $[9,10]$. Furthermore, antiPfs48/45 antibodies can be found in human sera from endemic areas, and correlate with TB activity [11-14]. Since pre-fertilization antigens are targets of the natural immune responses, immunity based on such antigens will have the added benefit of natural boosting. The two post-fertilization antigens Pfs 25 and Pfs 28 are lead targets for the development of TBVs, and are secreted onto the surface of ookinetes. Pfs 25 plays vital roles in ookinete survival in the midgut and penetration of the gut epithelium [15]. Mouse antiserum against native [16, 17], or heterologously expressed P25 inhibits parasite development in mosquitoes $[18,19]$. Currently, phase I human clinical trials using recombinant Pfs 25 and Pvs25 have demonstrated the production of antibodies that significantly inhibit transmission of the parasites, further highlighting their potential for TBV development [20, 21]. Recombinant Pvs25 expressed in yeast induces antibodies that block transmission by up to $80 \%$ in terms of mean oocyst intensity, and by $20-30 \%$ in reduction of prevalence of infection [20].

One important problem associated with TBV is that most recombinant candidate antigens such as Pfs 230 and Pfs $48 / 45$ require proper conformational folding of target epitopes to elicit functional antibodies [22]. DNA vaccine may overcome the need for such requirements associated with conventional protein immunization and has been shown to induce protective immune responses against several pathogens by eliciting both humoral and cellular immune responses [23-28]. DNA vaccines for Pfs25 have been shown to induce effective TB activity in mice and rhesus monkey [29-31]. Another problem is that most TBVs could not induce sterile TB activity to completely block the development of oocysts in mosquitoes. Thus, it is possible that combining two different TBV candidates that target both pre- and postfertilization antigens may improve TB activity. To test this hypothesis, we used the rodent malaria parasite Plasmodium yoelii as a model system and evaluated the immunogenicity and protective efficacy of DNA vaccines for Pys48/45 and Pys25. We show that these DNA vaccines can induce strong antibody response in mice, and the antibodies are functional in inhibiting zygote and ookinete formation in vitro and blocking oocyst formation in mosquitoes. However, simultaneous DNA immunization against both Pys 48 and Pys 25 did not achieve an additive effect in the induction of functional TB antibodies.

\section{Methods}

\section{Mice and parasites}

Female BALB/c mice aged from 6 to 8 weeks were used for vaccination and infection with the $P$. yoelii lethal strain 17XL (Py17XL). Infections with the Py17XL blood stages were initiated by intraperitoneal injection of $1 \times$ $10^{6}$ parasitized erythrocytes per mouse. Parasitemia was determined by microscopic examination of Giemsastained thin smears from the tail blood. Mortality of infected mice was recorded daily. All experiments were performed in compliance with the regulations of China Medical University Animal Ethics Committee.

\section{DNA constructs for immunization}

Genomic DNA from Py17XL was used for amplification of the Pys 48/45 and Pys25 gene. The Pys48/45 open reading frame (ORF) was amplified using primers $5^{\prime}$-AAG CTT ATG CTC TCC TTT TTT GGG-3' (HindIII site underlined) and 5'-GAT ATC TTA TAG CCA CAT AAA AA A TAA GGG AAT-3' (EcoRV site underlined). The Pys 25 ORF was PCR amplified with the primers $5^{\prime}$ GGA TCC ATG AAT ACT TAT TAC AGT GT-3' $\overline{(B a m} \mathrm{HI}$ site underlined) and 5'-CTC GAG TTA AAT GAT ATT TGA GAA TAA TAG-3' (XhoI site underlined). The PCR products were cloned into the DNA vaccine vector pcDNA3.1+ and the resultant plasmids were designated as Pys48/45-pcDNA3.1+ and Pys25-pcDNA3.1+, respectively. After verification of the inserts by sequencing, the plasmids were purified using the EndoFree Plasmid Maxi kit (Qiagen, Germany).

\section{Immunization scheme}

For DNA vaccine, plasmid DNA in $100 \mu \mathrm{l}$ of phosphatebuffered saline (PBS) was administered into the right and left tibialis cranialis muscles of the mouse by intramuscular immunizations. Mice were divided into five 
groups. Group 1 received $100 \mu \mathrm{l}$ of PBS as the negative control. Group 2, 3 and 4 received a dose of $50 \mu \mathrm{g}$ of empty vector pcDNA3.1+, Pys48/45-pcDNA3.1+ and Pys25-pcDNA3.1+, respectively. Group 5 received $50 \mu \mathrm{g}$ each of Pys48/45-pcDNA3.1+ and Pys25-pcDNA3.1+ in separate sites, which were not mixed in the same syringe. The injections were administered three times at a four-week interval. Four weeks after the first and each of the two subsequent booster immunizations, pooled sera were collected from all the mice for analysis by enzymelinked immunosorbent assay (ELISA). For each immunization scheme, five BALB/c mice were immunized.

\section{Preparation of gametocytes and ookinete culture}

The P. yoelii-infected blood was diluted in PBS to a hematocrit of $10 \%$ and layered onto a $45 \%$ (v/v) Percoll (Pharmacia GE) PBS cushion. After centrifugation at $350 \times g$ for $20 \mathrm{~min}$ at room temperature (RT), the gametocyte-enriched layer was collected at the interface and washed three times with PBS. Part of the purified gametocytes was then diluted 1:10 with RPMI 1640 medium supplemented with $50 \mu \mathrm{g} / \mathrm{ml}$ of hypoxanthine, 25 mM HEPES, 20 \% heat-inactivated fetal calf serum, $24 \mathrm{mM} \mathrm{NaHCO}, 5 \mathrm{U} / \mathrm{ml}$ penicillin, and $5 \mu \mathrm{g} / \mathrm{ml}$ streptomycin $(\mathrm{pH} 8.4)$ and cultured at $24{ }^{\circ} \mathrm{C}$ for $24 \mathrm{~h}$. The purified gametocyte and the cultured ookinete pellets were lysed by $2 \% \mathrm{SDS}$ in PBS at RT, then centrifuged at $12,000 \mathrm{rpm}$ for $10 \mathrm{~min}$ at $4{ }^{\circ} \mathrm{C}$. The protein extracts were stored at $-80{ }^{\circ} \mathrm{C}$ for ELISA and Western blotting.

\section{ELISA}

Serum samples obtained from immunized and control mice were tested for antibodies against Pys48 and Pys25 using ELISA as previously described [19]. Briefly, 96-well microtiter plates were pre-coated $(100 \mu \mathrm{l} /$ well $)$ with $10 \mu \mathrm{g}$ of purified gametocyte or cultured ookinete antigens in bicarbonate buffer at $4{ }^{\circ} \mathrm{C}$ overnight. For estimating endpoint titer of each immunized group, sera from all mice in each immunization group were pooled and diluted from 1:200 to 1:25600 in $1 \%$ bovine serum albumin (BSA) in PBS containing $0.05 \%$ Tween 20 (PBS-T). Plates were incubated for $2 \mathrm{~h}$, washed three times with PBS-T, and incubated with horseradish peroxidase (HRP)-conjugated goat anti-mouse IgG (1:5000) for $1 \mathrm{~h}$, followed by six washes with PBS-T. Finally, the optical density values were measured at $492 \mathrm{~nm} 20 \mathrm{~min}$ after the addition of the substrate.

\section{Western blot analysis}

Protein lysates from purified gametocytes or cultured ookinete pellets of Py17XL were separated by electrophoresis in a $10 \%$ SDS-polyacrylamide gel. Proteins were transferred to a $0.45 \mu \mathrm{m}$ PVDF membrane
(Millipore, USA). The membrane was blocked with $5 \%$ skimmed milk in Tris-buffered saline (TBS), and then incubated for $24 \mathrm{~h}$ at $4{ }^{\circ} \mathrm{C}$ with pooled mouse antisera at 1:100 in TBS containing $0.1 \%$ Tween 20 (TBST). After three washes with TBST, the membrane was incubated for $1 \mathrm{~h}$ with HRP-conjugated goat anti-mouse IgG (Proteintech $^{\mathrm{m}}$, USA) diluted 1:10,000 in TBST. After three washes with TBST, the proteins were visualized with ECL Western Blotting Substrate (Thermo Pierce, USA) and detected using the BioImaging System (Tanon, China). The relative molecular masses of proteins were estimated with PageRuler ${ }^{\mathrm{Tm}}$ Prestained Protein Ladder (10-170 kDa) (Fermentas, USA).

\section{Indirect immunofluorescence assay (IFA)}

Purified gametocyte or cultured ookinetes were spotted onto multi-well slides, air-dried, and fixed with ice-cold acetone for IFA. Slides were first blocked with PBS containing $5 \%$ skimmed milk for $30 \mathrm{~min}$ at $37{ }^{\circ} \mathrm{C}$ and then incubated with anti-Pys 48 or anti-Pys 25 mouse sera (1:50) for $1 \mathrm{~h}$ at $37^{\circ} \mathrm{C}$. After rinsing with PBS, the slides were incubated with fluorescein isothiocyanate (FITC)conjugated goat anti-mouse IgG antibodies (Tago, Camarillo, CA) and the nuclear stain 4',6-diamidino-2phenylindole (DAPI) for $30 \mathrm{~min}$ at $37^{\circ} \mathrm{C}$. After rinsing with PBS, the slides were mounted under a coverslip in bicarbonate-buffered glycerin, and observed under a fluorescence microscope.

\section{In vitro zygote and ookinete development assay}

To examine whether immune sera possessed TB activity, in vitro zygote and ookinete conversion assay was performed [32]. Ten $\mu$ l of $P$. yoelii-infected blood was taken from mouse tails on day 3 post-infection and mixed with $90 \mu \mathrm{l}$ of complete ookinete culture medium containing $20 \mu \mathrm{l}$ of control or immune sera (1:4). The cultures were incubated at $24{ }^{\circ} \mathrm{C}$ for $24 \mathrm{~h}$. Parasites were harvested by centrifugation $(500 \times g, 5 \mathrm{~min})$ and the pellet resuspended in $40 \mu \mathrm{l}$ of PBS. One $\mu \mathrm{l}$ of the suspension was placed on a slide for fluorescent microscopy and blocked with $5 \%$ fat-free milk for $1 \mathrm{~h}$ at $37^{\circ} \mathrm{C}$. After rinsing with PBS, the slides were incubated with Pys 25 monoclonal antibodies (mAbs) at 1:200 for $1 \mathrm{~h}$, followed by FITCconjugated goat anti-mouse IgG for $1 \mathrm{~h}$. The total numbers of zygotes and ookinetes formed per microlitre of the aliquots were counted.

\section{Mosquito feeding experiment}

Four weeks after the last immunization, mice from each of the five groups were inoculated with $1 \times 10^{6}$ Py17XLparasitized erythrocytes/mouse. Three days after infection, three mice from each group were used for mosquito feeding experiments. Four-day-old female Anopheles stephensi mosquitoes were starved overnight 
and then allowed to feed on Py17XL-infected mice (50 mosquitoes/mouse) for $30 \mathrm{~min}$. The engorged mosquitoes were separated and maintained at $24{ }^{\circ} \mathrm{C}$ on $1.5 \%$ fructose and $1.5 \%$ sucrose. Nine days after feeding, mosquitoes were dissected to count infected mosquitoes (prevalence of infection) and oocyst density (number of oocysts/infected midgut) by microscopy. Oocyst density was derived from the dissection of 20-30 mosquitoes per mouse.

\section{Statistical analysis}

Statistical analysis of ELISA data and in vitro TB activity of the immune sera was performed by the GraphPad Prism software. For the ELISA data, one-way ANOVA was used to compare all the immunized groups. The formation of P. yoelii zygotes and ookinetes was analyzed by the Mann-Whitney U-Test. Considering the overdispersion nature of the oocyst density and prevalence distribution, these data were analyzed by using a zeroinflated Generalized Linear Mixed-Model statistical model (GLMM) [33]. A value of $P<0.05$ was considered significant.

\section{Results}

\section{Antibody responses to DNA vaccines}

Current TBV designs target pre-fertilization antigens such as P48/45 or post-fertilization antigens such as the major ookinete surface antigen P25. To test whether a combination vaccine targeting both pre- and postfertilization antigens would perform better, we tested the combination of Pys48/45 and Pys 25 using the rodent malaria parasite $P$. yoelii as a model. DNA vaccine constructs were used in order to circumvent difficulties associated with producing correctly folded recombinant proteins of the two Cys-motif proteins Pys48/45 and Pys25 in prokaryotic expression systems. Groups of five mice were immunized with DNA vaccines against either individual antigens (Pys48/45 or Pys25) or combination antigens (both Pys48/45 and Pys25). Antibody responses were measured by ELISA with whole cell lysates of purified gametocytes or cultured ookinetes. Compared with mice in the control group, immunization of mice with the empty pcDNA3.1+ vector did not produce any noticeable antibody response against parasite sexual stage antigens. Consistent with the abundant expression of Pys 48/45 in gametocytes and Pys25 in ookinetes, immunization with Pys48/45-pcDNA3.1+ individually and in combination with Pys25-pcDNA3.1+ produced significantly higher antibody titers to the gametocyte lysate than the empty vector, whereas immunization with Pys25-pcDNA3.1+ individually and in combination with Pys48/45-pcDNA3.1+ produced significant higher antibody responses to the ookinete lysate than the empty vector (Fig. 1a, b). The antibody production showed a significant increase in each immunized mouse after the first and second boost (Fig. 1a, b). These results suggested that mice immunized with both DNA vaccine constructs produced specific antibodies against either gametocyte or ookinete antigen(s). Intriguingly, vaccination with both constructs simultaneously resulted in

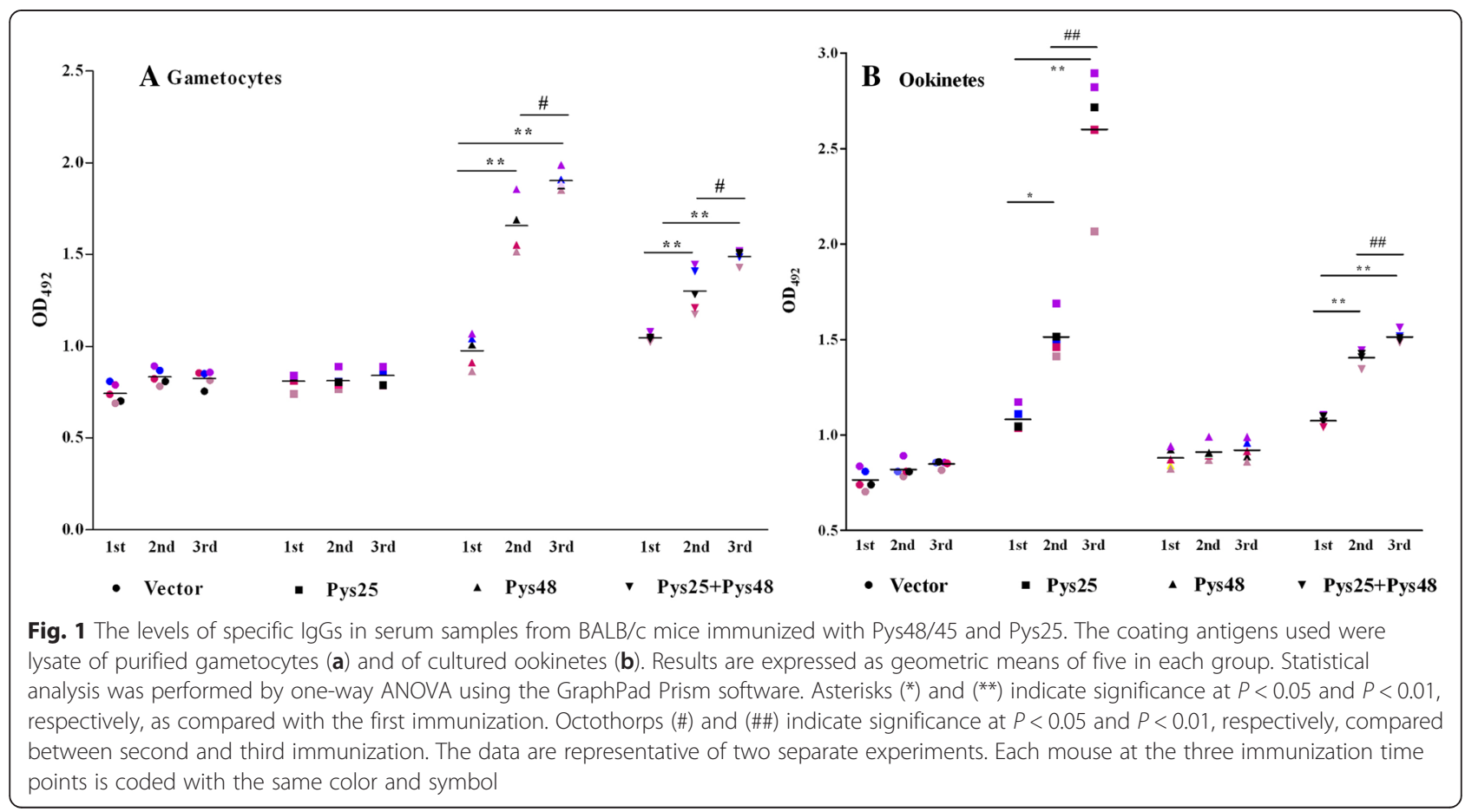


lower antibody titers than those immunized with the two constructs individually (Fig. 1).

\section{Reactivity of the immune sera with Pys48/45 and Pys25 proteins}

In order to confirm that the specific antibodies produced against gametocyte and ookinete lysates were indeed specific for Pys48/45 and Pys25, respectively, we performed Western blot and IFA analyses. Western blots showed that the immune sera from Pys48/45-pcDNA3.1 + vaccinated mice detected an approximately $45 \mathrm{kDa}$ protein in the gametocyte lysate, whereas the immune sera from Pys25-pcDNA3.1+ vaccinated mice detected a $\sim 25 \mathrm{kDa}$ protein in the ookinete lysate (Fig. 2), indicating that these antisera specifically recognized Pys $48 / 45$ and Pys25, respectively. Sera from combination antigens (both Pys48/45 and Pys25) immunized group detected both the $45 \mathrm{kDa}$ protein and the $25 \mathrm{kDa}$ protein, indicating that the antisera recognized both Pys48/45 and Pys25 (Fig. 2). Although there were minor cross-reacting bands in the Western blot with the gametocyte lysates, the dominant bands were consistent with the predicted molecular weight of the Pys48/45. Furthermore, IFA demonstrated that these immune sera reacted predominantly with the surface of Py17XL gametocytes and ookinetes, respectively (Fig. 3).

\section{In vitro TB activity of the immune sera}

To assess TB activity of the immune sera from the DNA vaccines, in vitro zygote and ookinete inhibition assay was performed using these immune sera at 1:4 dilution. Zygote and ookinete formation was quantified using IFA to detect the major ookinete surface antigen Pys25. Compared with the control sera, sera from the vector control group did not have any inhibitory activity on zygote and ookinete formation (Table 1). In contrast, the three immunization groups all exhibited significantly higher inhibitory activity on zygote formation than the control group (ANOVA: $F_{(3,12)}=194.6, P<0.0001$ ). The numbers of zygotes formed with sera from the three immunization groups were reduced by at least 7 -fold compared with the control groups (Table 1). Similarly, incubation with sera from the immunization groups resulted in at least 23 -fold reduction in the number of ookinetes (Table 1). The best inhibitory activity was from the Pys25-pcDNA3.1+ vaccination group, where zygote formation was reduced by $\sim 17$-fold and ookinete development was completely blocked (ANOVA: $F_{(3,12)}=$ 74.67, $P<0.0001)$. Consistent with the observed lower parasite-specific antibody titers induced with the combination immunization, sera from mice immunized with Pys48/45-pcDNA3.1+ and Pys25-pcDNA3.1+ together did not show higher inhibitory activity on zygote and ookinete formation than sera immunized with these constructs individually (Table 1).

\section{TB activity in mosquito feeding experiments}

To compare the TB activity of different immunization schemes, An. stephensi mosquitoes were used to feed on mice in the different immunization groups on day 3 after Py17XL infection. Mosquitoes were dissected on day 9 after feeding to determine the mosquito infection rate and oocyst density. In the control group, $100 \%$ mosquitoes were infected with median oocyst density exceeding

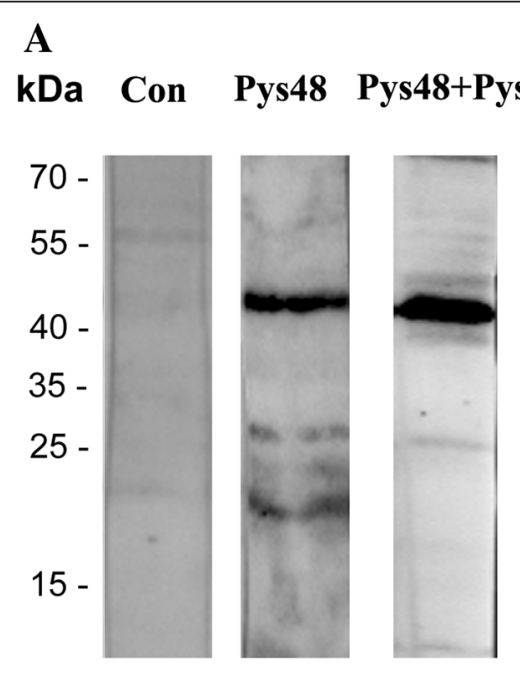

Gametocytes lysate
B
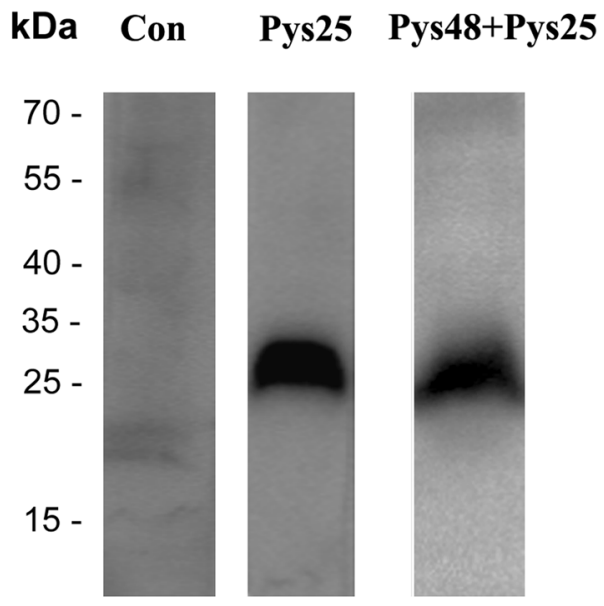

\section{Ookinetes lysate}

Fig. 2 Western blot analysis of Pys48/45 and Pys 25 expression in P. yoelii gametocytes and ookinetes. Lysates from gametocyte- (a) and ookineteenriched (b) preparations were probed with immune sera from the Pys48/45, Pys 25 and Pys48/45 + Pys25 DNA immunization groups, respectively 


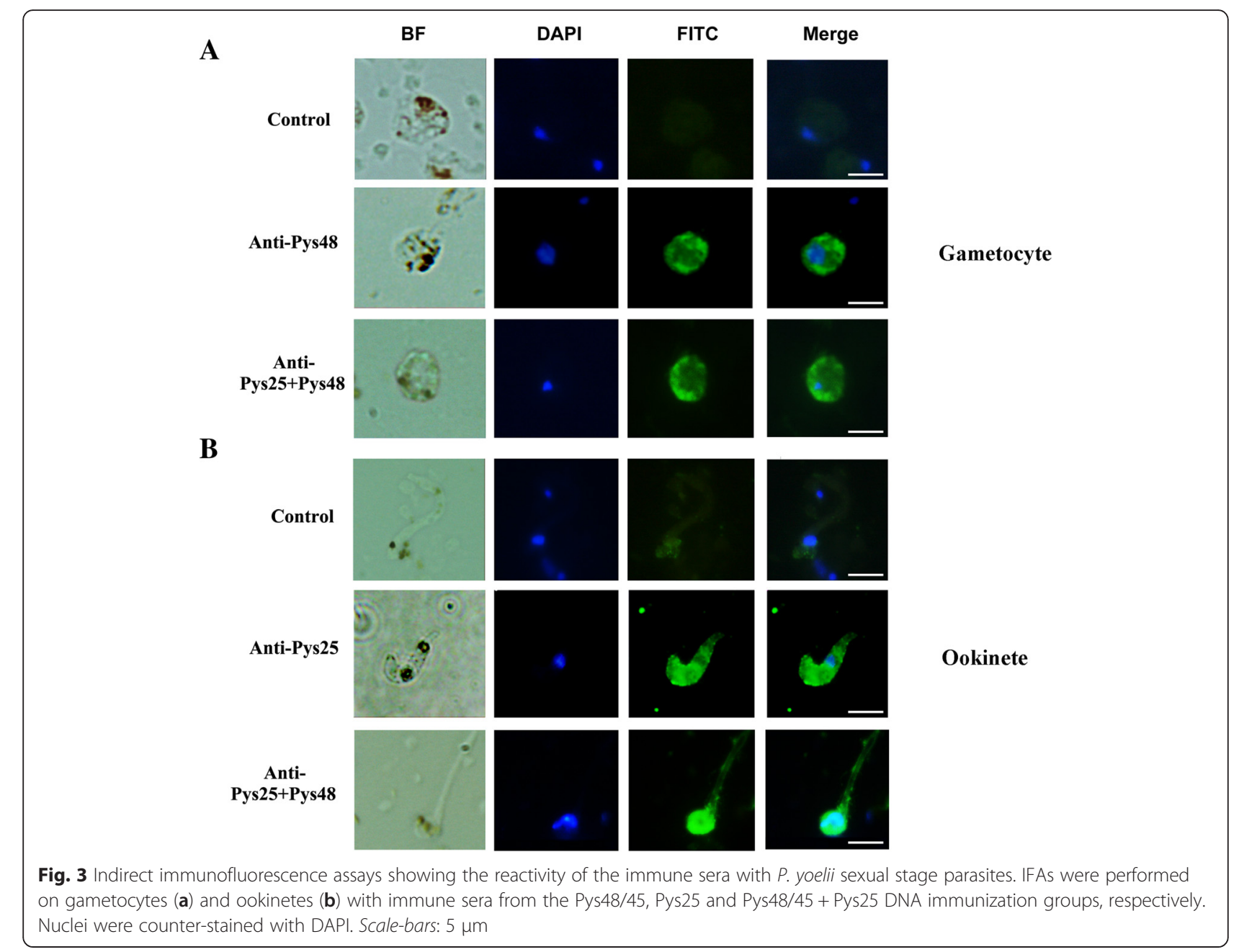

200 oocysts per midgut. In comparison, mice receiving the DNA vaccines Pys48/45-pcDNA3.1+ and Pys25-pcDNA3.1 + either individually or in combination significantly reduced the prevalence of infected mosquitoes and oocyst density $(P<0.001$, Fig. 4). Compared with $100 \%$ infectivity in the control group, immunization with Pys25-pcDNA3.1+,

Table 1 Inhibitory activity of immune sera from the DNA vaccines on the in vitro formation of $P$. yoelii zygotes and ookinetes

\begin{tabular}{llll}
\hline Group & \multicolumn{2}{l}{ Median no. of parasites/well (range) } & \multirow{2}{*}{$\begin{array}{c}\text { \% inhibition } \\
\text { of ookinetes }\end{array}$} \\
\cline { 2 - 3 } & Zygotes & Ookinetes & \\
\hline Naïve mice & $45.0(39-51)$ & $13.5(8-17)$ & \\
Vector control & $42.0(37-47)$ & $11.5(9-14)$ & 14.8 \\
Pys25 & $2.5(1-4)^{\mathrm{a}}$ & $0.0(0-1)^{\mathrm{a}}$ & 100 \\
Pys48 & $4.5(3-6)^{\mathrm{a}}$ & $0.5(0-1)^{\mathrm{a}}$ & 96.3 \\
Pys25 + Pys48 & $6.0(4-8)^{\mathrm{a}}$ & $0.5(0-1)^{\mathrm{a}}$ & 96.3 \\
\hline
\end{tabular}

${ }^{\text {a }}$ Significant difference compared with the Naïve mice group at $P<0.05$ by the Mann-Whitney U-Test
Pys48/45-pcDNA3.1+ and their combination led to 40.7, 28.6 , and $14.3 \%$ reduction in the prevalence of infected mosquitoes, respectively. In addition, the average number of oocysts per midgut was reduced to $3,23.5$ and 18.5 oocysts/ midgut in these immunization groups, respectively (Table 2). Again, immunization with Pys25-pcDNA3.1+ produced the best TB activity. Further, the combination immunization group, although appeared slightly better than the Pys48/45pcDNA3.1+ immunization group, showed lower TB activity than Pys25-pcDNA3.1+ single immunization in both the prevalence of infection and oocyst density.

\section{Discussion}

Due to the complex nature of the malaria parasite's life cycle, the development of multi-stage vaccines targeting the major stages (pre-erythrocytic, asexual blood and sexual stages) is a more effective vaccination strategy. In addition, mosquito midgut proteins are alternative TBV candidates [34]. Immune responses to individual immunogens in vaccine cocktails have been observed [35, 36], 


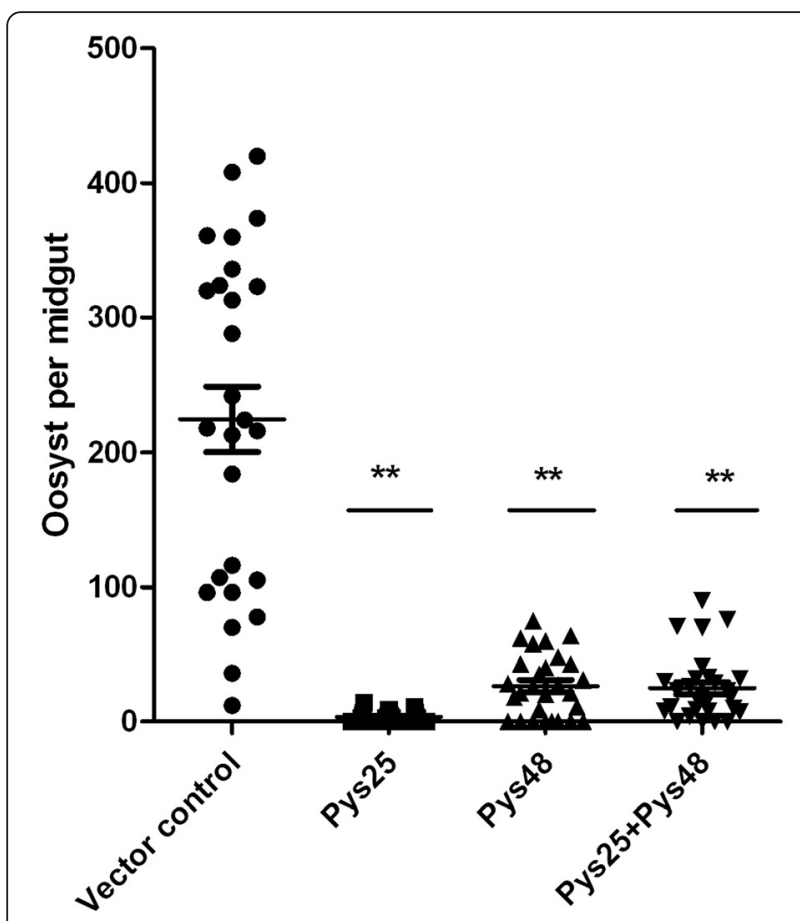

Fig. 4 Transmission-blocking effect of immunization with Pys25, Pys48/45 and Pys48/45 + Pys 25 plasmids in direct mosquito feeding assay. Data points represent the number of oocysts in individual mosquitoes, horizontal bars indicate the mean number of oocysts per midgut and error bars indicate SEM within individual treatments.

Three independent experiments were performed. Asterisk (**) indicates significance at $P<0.01$ as compared with the Vector control

and vaccines targeting more antigens or epitopes may provide better protection than those for single antigens or epitopes in animal models [37-39]. Vaccines targeting antigens of the same stage are expected to have a synergistic effect, however, this may not always be the case. For example, antibodies to Pfs25 and Pfs28 were found to have synergistic TB activity [40], whereas antibodies to the orthologous Pvs25 and Pvs28 did not show obvious synergism [41]. Given that blocking individual stages requires different stage-specific antibody titers, combination of antigens in cocktail vaccines may need to be evaluated on a case-by-case basis [42].

DNA-based vaccines have been shown to generate both cellular and humoral immune responses in diverse animal models. In this study, we evaluated the TB effect of combination DNA vaccines against two sexual-stage antigens in a rodent malaria model. The two antigens tested, P25 and P48/45, are both leading TBV candidates. Since the immunogenicity of a vaccine depends on the formation of natural conformational epitopes, and it is especially important for P48 which contains a unique arrangement of six cysteine-containing domains [43], we expect that DNA vaccine would circumvent the difficulties in obtaining conformationally correct P48 [44] as shown for the Pvs48/45 [45]. Consistent with DNA vaccine results for the Pfs25 [29] as well as the Pvs48/45 [45], DNA vaccines with the Pys25 and Pys48/ 45 individually and in combination produced evident, specific antibody responses, and the antibodies recognized the respective native proteins in parasites and possessed significant TB activity. In both in vitro ookinete conversion and direct mosquito feeding assays, immunization with the Pys 25 plasmid produced much better TB activity than with the Pys48/45 construct, which agrees with P25 being one of the best TBV candidates in numerous experiments $[22,46]$. As in a vaccination study with recombinant Pfs25-Pfs230, immune responses are strongly biased towards Pfs 25 [47]. This could be due to a difference in the distribution and immunogenicity of the B epitopes, which may be inherent characteristics of individual proteins. More surprisingly, immunization with both DNA constructs produced decreased humoral responses to each of the two antigens as compared with immunization with individual DNA constructs, suggesting of interference between the two DNA vaccine constructs. This phenomenon is consistent with an earlier report showing a similar interference effect of DNA vaccination with Pfs25 and the gametocyte

Table 2 Transmission-blocking effect anti-Pys 25 and Pys48/45 sera in direct mosquito feeding assay

\begin{tabular}{|c|c|c|c|c|}
\hline & Vector control & Pys25 & Pys48 & Pys $25+$ Pys 48 \\
\hline $\mathrm{N}$ & 26 & 27 & 28 & 28 \\
\hline \# not infected & 0 & 11 & 8 & 4 \\
\hline Prevalence (\%) ${ }^{\mathrm{a}}$ & 100.0 & 59.3 & 71.4 & 85.7 \\
\hline Median & 221.0 & 3.0 & 23.5 & 18.5 \\
\hline Mean & 224.6 & 3.6 & 26.4 & 24.9 \\
\hline Range & 420 & 14 & 75 & 71 \\
\hline Reduction in Oocyst (\%) & na & 98.6 & 89.4 & 91.6 \\
\hline Reduction in Prevalence $(\%)^{c}$ & na & 40.7 & 28.6 & 14.3 \\
\hline
\end{tabular}

${ }^{a}$ The prevalence of infection was calculated by number of mosquitoes with oocysts/total mosquitoes dissected in each group

$\mathrm{b}_{\%}$ reduction in oocyst was calculated as $\left(\right.$ median $_{\text {control }}-$ median $_{\text {pcDNA3.1 }}+$ plasmid $) /$ median $_{\text {control }} \times 100$

$c_{\%}$ reduction in prevalence was calculated as $\left(\%\right.$ prevalence control $\%$ prevalence pcDNA3.1 $\left._{\text {plasmid }}\right) / \%$ prevalence control $\times 100$ 
antigen Pfg27, which showed lower TB activity in the combination vaccination group [29]. Alternatively, the reduced antibody responses to individual antigens produced in the mixed vaccination scheme could be a dosage effect, as the doubled amount of DNA used for combination immunization may interfere with antigen presentation.

\section{Conclusion}

The data described here indicate that both Pys 25 and Pys48/45 DNA vaccines showed remarkable immunogenicity and induced functional TB activity. Though both antigens are considered leading TBV candidates targeting pre- and post-fertilization antigens respectively, their combination in immunization produced an interfering effect in eliciting immune responses to either protein. It remains to be tested whether this is a phenomenon restricted to DNA vaccine only. Nonetheless, these results could have important implications in the design of future multicomponent DNA vaccines.

\section{Abbreviations \\ BSA, bovine serum albumin; DAPI, 4',6-diamidino-2-phenylindole; ELISA, enzyme-linked immunosorbent assay; FITC, fluorescein isothiocyanate; GLMM, Generalized Linear Mixed-Model statistical model; HRP, horseradish peroxidase; IFA, Indirect immunofluorescence assay; ORF, open reading frame; PBS, phosphate-buffered saline; Py17XL, P. yoelii lethal strain 17XL; RT, room temperature; TB, Transmission-blocking; TBS, Tris-buffered saline; TBV, Transmission-blocking vaccine}

\section{Acknowledgements \\ We thank Dr. Thomas S. Churcher (Department of Infectious Disease Epidemiology, Imperial College London), who provided us the statistical program. We thank Dr. Lianfeng Shan in China Medical University for statistical support. This work was supported by grants from the National Institutes of Health, USA (R01Al099611-01) and National Natural Science Foundation of China (30972774).}

\section{Funding}

This work was supported by grants from the National Institutes of Health USA (R01Al099611-01) and National Natural Science Foundation of China (30972774).

\section{Availability of data and material}

The datasets supporting the conclusions of this article are included within the article.

\section{Authors' contributions}

YC and LC conceived the study and helped draft the manuscript. LZ carried out the studies, statistical analysis and drafted the manuscript. WP took part in the construction of DNA plasmids and purified the plasmids. ZQ and EL participated in the antibody detection and transmission blocking assay. All authors contributed to the writing of the manuscript and approved the submitted version of the manuscript.

\section{Authors' information}

Li Zheng: Associate Professor, Department of Immunology, College of Basic Medical Sciences, China Medical University.

Wei Pang: Lecturer, Department of Immunology, College of Basic Medical Sciences, China Medical University.

Zanmei Qi: Professor, Department of Immunology, College of Basic Medical Sciences, China Medical University.

Enjie Luo: Professor, Department of Pathogen Biology, College of Basic Medical Sciences, China Medical University.
Liwang Cui: Professor, Department of Entomology, The Pennsylvania State University.

Yaming Cao: Professor, Department of Immunology, College of Basic Medical Sciences, China Medical University.

\section{Competing interests}

The authors declare that they have no competing interests.

Consent for publication

Not applicable.

Ethics approval and consent to participate

Animal use was carried out following the guidelines of the animal ethics committee of China Medical University.

\section{Author details}

'Department of Immunology, College of Basic Medical Sciences, China Medical University, No.77 Puhe Road, Shenyang North New Area, Shenyang, Liaoning Province, People's Republic of China. ${ }^{2}$ Department of Pathogen Biology, College of Basic Medical Sciences, China Medical University, Shenyang, Liaoning, 110001, China. ${ }^{3}$ Department of Entomology, The Pennsylvania State University, 501 ASI Bldg., University Park, PA 16802, USA.

Received: 5 January 2016 Accepted: 19 July 2016

Published online: 08 August 2016

\section{References}

1. WHO. World malaria report. 2015. http://www.who.int/malaria/publications/ world-malaria-report-2015/report/en/.

2. Banek K, Lalani M, Staedke SG, Chandramohan D. Adherence to artemisininbased combination therapy for the treatment of malaria: a systematic review of the evidence. Malar J. 2014;13:7.

3. Yakasai AM, Hamza M, Dalhat MM, Bello M, Gadanya MA, Yaqub ZM, Ibrahim DA, Hassan-Hanga F. Adherence to artemisinin-based combination therapy for the treatment of uncomplicated malaria: a systematic review and meta-analysis. J Trop Med. 2015;2015:189232.

4. Weiss GE, Crabb BS, Gilson PR. Overlaying molecular and temporal aspects of malaria parasite invasion. Trends Parasitol. 2016;32(4):284-95.

5. Hoffman SL, Vekemans J, Richie TL, Duffy PE. The march toward malaria vaccines. Am J Prev Med. 2015;49(6 Suppl 4):S319-33.

6. Dimopoulos G, Kafatos FC, Waters AP, Sinden RE. Malaria parasites and the anopheles mosquito. Chem Immunol. 2002;80:27-49.

7. Carter R, Mendis KN, Miller LH, Molineaux L, Saul A. Malaria transmissionblocking vaccines - how can their development be supported? Nat Med. 2000;6(3):241-4.

8. van Dijk MR, Janse CJ, Thompson J, Waters AP, Braks JA, Dodemont HJ, et al. A central role for P48/45 in malaria parasite male gamete fertility. Cell. 2001;104(1):153-64.

9. Roeffen W, Teelen K, van As J, vd Vegte-Bolmer M, Eling W, Sauerwein R. Plasmodium falciparum: production and characterization of rat monoclonal antibodies specific for the sexual-stage Pfs48/45 antigen. Exp Parasitol. 2001; 97(1):45-9.

10. Vermeulen AN, Roeffen WF, Henderik JB, Ponnudurai T, Beckers PJ, Meuwissen JH. Plasmodium falciparum transmission blocking monoclonal antibodies recognize monovalently expressed epitopes. Dev Biol Stand. 1985;62:91-7.

11. Bousema JT, Drakeley CJ, Kihonda J, Hendriks JC, Akim NI, Roeffen W, Sauerwein RW. A longitudinal study of immune responses to Plasmodium falciparum sexual stage antigens in Tanzanian adults. Parasite Immunol. 2007;29(6):309-17.

12. Bousema JT, Roeffen W, van der Kolk M, de Vlas SJ, van de Vegte-Bolmer M, Bangs MJ, et al. Rapid onset of transmission-reducing antibodies in javanese migrants exposed to malaria in papua, indonesia. Am J Trop Med Hyg. 2006;74(3):425-31.

13. Roeffen W, Lensen T, Mulder B, Teelen K, Sauerwein R, Van Druten J, et al. A comparison of transmission-blocking activity with reactivity in a Plasmodium falciparum 48/45-kD molecule-specific competition enzymelinked immunosorbent assay. Am J Trop Med Hyg. 1995;52(1):60-5.

14. Ouedraogo AL, Roeffen W, Luty AJ, de Vlas SJ, Nebie I, Ilboudo-Sanogo E, et al. Naturally acquired immune responses to Plasmodium falciparum 
sexual stage antigens Pfs48/45 and Pfs230 in an area of seasonal transmission. Infect Immun. 2011;79(12):4957-64.

15. Tomas AM, Margos G, Dimopoulos G, van Lin LH, de Koning-Ward TF, Sinha R, et al. P25 and P28 proteins of the malaria ookinete surface have multiple and partially redundant functions. EMBO J. 2001;20(15):3975-83.

16. Tirawanchai N, Sinden RE. Three non-repeated transmission blocking epitopes recognized in the $21 \mathrm{kD}$ surface antigen of zygotes-ookinetes of Plasmodium berghei. Parasite Immunol. 1990;12(4):435-46.

17. Tirawanchai N, Winger LA, Nicholas J, Sinden RE. Analysis of immunity induced by the affinity-purified 21-kilodalton zygote-ookinete surface antigen of Plasmodium berghei. Infect Immun. 1991;59(1):36-44.

18. Kaslow DC, Bathurst IC, Lensen T, Ponnudurai T, Barr PJ, Keister DB. Saccharomyces cerevisiae recombinant Pfs 25 adsorbed to alum elicits antibodies that block transmission of Plasmodium falciparum. Infect Immun. 1994:62(12):5576-80.

19. Hisaeda H, Stowers AW, Tsuboi T, Collins WE, Sattabongkot JS, Suwanabun N, et al. Antibodies to malaria vaccine candidates Pvs 25 and Pvs28 completely block the ability of Plasmodium vivax to infect mosquitoes. Infect Immun. 2000; 68(12):6618-23.

20. Malkin EM, Durbin AP, Diemert DJ, Sattabongkot J, Wu Y, Miura K, et al. Phase 1 vaccine trial of Pvs25H: a transmission blocking vaccine for Plasmodium vivax malaria. Vaccine. 2005;23(24):3131-8.

21. Wu Y, Ellis RD, Shaffer D, Fontes E, Malkin EM, Mahanty S, et al. Phase 1 trial of malaria transmission blocking vaccine candidates Pfs 25 and Pvs25 formulated with montanide ISA 51. PLoS One. 2008;3(7):e2636.

22. Kapulu MC, Da DF, Miura K, Li Y, Blagborough AM, Churcher TS, et al. Comparative assessment of transmission-blocking vaccine candidates against Plasmodium falciparum. Sci Rep. 2015;5:11193.

23. Watts AM, Kennedy RC. DNA vaccination strategies against infectious diseases. Int J Parasitol. 1999;29(8):1149-63.

24. Gurunathan S, Klinman DM, Seder RA. DNA vaccines: immunology, application, and optimization*. Annu Rev Immunol. 2000;18:927-74.

25. Liu MA. DNA vaccines: an historical perspective and view to the future. Immunol Rev. 2011:239(1):62-84.

26. Mallilankaraman $K$, Shedlock DJ, Bao H, Kawalekar OU, Fagone P, Ramanathan $A A$, et al. A DNA vaccine against chikungunya virus is protective in mice and induces neutralizing antibodies in mice and nonhuman primates. PLoS Negl Trop Dis. 2011;5(1):e928.

27. Oosterhuis $K$, van den Berg JH, Schumacher TN, Haanen JB. DNA vaccines and intradermal vaccination by DNA tattooing. Curr Top Microbio Immunol. 2012;351:221-50.

28. Lin F, Shen X, McCoy JR, Mendoza JM, Yan J, Kemmerrer SV, et al. A novel prototype device for electroporation-enhanced DNA vaccine delivery simultaneously to both skin and muscle. Vaccine. 2011;29(39):6771-80.

29. Lobo CA, Dhar R, Kumar N. Immunization of mice with DNA-based Pfs 25 elicits potent malaria transmission-blocking antibodies. Infect Immun. 1999; 67(4):1688-93.

30. Coban C, Philipp MT, Purcell JE, Keister DB, Okulate M, Martin DS, et al. Induction of Plasmodium falciparum transmission-blocking antibodies in nonhuman primates by a combination of DNA and protein immunizations. Infect Immun. 2004;72(1):253-9.

31. Kumar R, Nyakundi R, Kariuki T, Ozwara H, Nyamongo O, Mlambo G, et al. Functional evaluation of malaria Pfs25 DNA vaccine by in vivo electroporation in olive baboons. Vaccine. 2013;31(31):3140-7.

32. Reininger $L$, Billker $O$, Tewari $R$, Mukhopadhyay A, Fennell C, Dorin-Semblat $D$, et al. A NIMA-related protein kinase is essential for completion of the sexual cycle of malaria parasites. J Biol Chem. 2005;280(36):31957-64.

33. Churcher TS, Blagborough AM, Delves M, Ramakrishnan C, Kapulu MC, Williams AR, et al. Measuring the blockade of malaria transmission - an analysis of the Standard Membrane Feeding Assay. Int J Parasitol. 2012; 42(11):1037-44.

34. Lavazec C, Bourgouin C. Mosquito-based transmission blocking vaccines for interrupting Plasmodium development. Microbes Infect. 2008;10(8):845-9.

35. Bathurst IC, Gibson HL, Kansopon J, Hahm BK, Green KM, Chang SP, et al. An experimental vaccine cocktail for Plasmodium falciparum malaria. Vaccine. 1993;11(4):449-56.

36. Boes A, Spiegel H, Voepel N, Edgue G, Beiss V, Kapelski S, et al. Analysis of a multi-component multi-stage malaria vaccine candidate - tackling the cocktail challenge. PLoS One. 2015;10(7):e0131456.
37. Khusmith S, Charoenvit Y, Kumar S, Sedegah M, Beaudoin RL, Hoffman SL. Protection against malaria by vaccination with sporozoite surface protein 2 plus CS protein. Science. 1991;252(5006):715-8.

38. Patarroyo ME, Romero P, Torres ML, Clavijo P, Moreno A, Martinez A, et al. Induction of protective immunity against experimental infection with malaria using synthetic peptides. Nature. 1987;328(6131):629-32.

39. Tine JA, Lanar DE, Smith DM, Wellde BT, Schultheiss P, Ware LA, et al. NYVAC-Pf7: a poxvirus-vectored, multiantigen, multistage vaccine candidate for Plasmodium falciparum malaria. Infect Immun. 1996;64(9):3833-44.

40. Duffy PE, Kaslow DC. A novel malaria protein, Pfs28, and Pfs 25 are genetically linked and synergistic as falciparum malaria transmissionblocking vaccines. Infect Immun. 1997;65(3):1109-13.

41. Hisaeda H, Collins WE, Saul A, Stowers AW. Antibodies to Plasmodium vivax transmission-blocking vaccine candidate antigens Pvs25 and Pvs28 do not show synergism. Vaccine. 2001;20(5-6):763-70.

42. Spiegel H, Boes A, Kastilan R, Kapelski S, Edgue G, Beiss V, et al. The stagespecific in vitro efficacy of a malaria antigen cocktail provides valuable insights into the development of effective multi-stage vaccines. Biotechnol J. 2015;10(10):1651-9.

43. Arredondo SA, Cai M, Takayama Y, MacDonald NJ, Anderson DE, Aravind $L$, et al. Structure of the Plasmodium 6-cysteine s48/45 domain. Proc Natl Acad Sci U S A. 2012;109(17):6692-7.

44. Donnelly JJ, Ulmer JB, Shiver JW, Liu MA. DNA vaccines. Annu Rev Immunol. 1997;15:617-48

45. Tachibana M, Suwanabun N, Kaneko O, Iriko H, Otsuki H, Sattabongkot J, et al. Plasmodium vivax gametocyte proteins, Pvs48/45 and Pvs47, induce transmission-reducing antibodies by DNA immunization. Vaccine. 2015; 33(16):1901-8.

46. Kumar R, Ray PC, Datta D, Bansal GP, Angov E, Kumar N. Nanovaccines for malaria using Plasmodium falciparum antigen Pfs25 attached gold nanoparticles. Vaccine. 2015;33(39):5064-71.

47. Beiss V, Spiegel H, Boes A, Kapelski S, Scheuermayer M, Edgue G, et al. Heatprecipitation allows the efficient purification of a functional plant-derived malaria transmission-blocking vaccine candidate fusion protein. Biotechnol Bioeng. 2015;112(7):1297-305.

\section{Submit your next manuscript to BioMed Central and we will help you at every step:}

- We accept pre-submission inquiries

- Our selector tool helps you to find the most relevant journal

- We provide round the clock customer support

- Convenient online submission

- Thorough peer review

- Inclusion in PubMed and all major indexing services

- Maximum visibility for your research

Submit your manuscript at www.biomedcentral.com/submit
Biomed Central 